\title{
Flywheel energy storage control system with the system operating status control via the Internet
}

\author{
MARCIN BASZYŃSKI, TOMASZ SIOSTRZONEK \\ Department of Power Electronics and Energy Control Systems \\ AGH, University of Science and Technology \\ e-mail:mbaszyn@agh.edu.pl,tsios@agh.edu.pl
}

(Received: 10.03.2014, revised: 26.06.2014)

\begin{abstract}
Modern electronics systems consist of not only with the power electronics converters, but also with the friendly user interface which allow you to read the operating parameters and change them. The simplest solution of the user interface is to use alphanumeric display which displays information about the state of the converter. With a few additional buttons you can change the settings. This solution is simple, inexpensive but allows only local control (within walking distance from the system) and the number of displayed information is low. You can create extensive menu, but it causes problems with access to information. This paper presents the example of a rotating energy storage universal solution which is lack of the above mentioned disadvantages
\end{abstract}

Key word: BLDCPM, high speed drive, flywheel energy storage system, FPGA

\section{Introduction}

Flywheel energy storages systems (FESS) are modern mechatronic systems with a wide range of applications [1]:

- in systems improving the quality of electrical energy in order to reduce the impact of momentary active power surges on the supply network as well as to mitigate peak loads;

- in redundant supply systems for operation continuity or actuating other electrical energy sources;

- in accumulation and controlled output systems of energy generated by alternative standalone electrical energy sources, such as photovoltaic power plants or wind farms;

- in wind farm power fluctuation reduction;

- in the storage of the energy recovered during regenerative braking of frequently started and stopped drives;

- in applications for lift operation in buildings with a high traffic load ("intelligent building"); 
- in large industrial plants (high power accumulators) in order to reduce voltage fluctuation;

- for supplying receivers requiring high portions of energy transmitted in a short time.

Regardless of the chosen system operating parameter control method, it is important that the user interface that enables changing the device status and parameters is clear and userfriendly. The development of mobile platforms has resulted in the appearance on the market of a large quantity of inexpensive devices with graphic touch displays. These devices are equipped with a USB communication interface and WiFi and Bluetooth wireless communication modules. The mobile device market is shared by three leading operating systems: Windows, iOS and Android. The last one has gained the most advantage over the other two owing to the documentation availability.

The combination of a control system with the Internet enables the parameter control and change of operating parameters from any location in the world with the Internet access. At current technology development rate, there are numerous methods to execute this task, e.g. through:

- HTTP server implementation within a microsystem. An advantage of this solution is easy access to information from the control system via any web browser, which makes it a solution independent of the hardware and the operating system of the device from which the web page is displayed. Unfortunately, this approach requires an individual public IP address to be assigned to the controller. The incorporation of the controller into the Internet via, e.g., a router restricts the access to it only to the devices using this router. The controller is then assigned an IP address from the pool available for the router and is not visible from the external network. Since the server is accessible for all users within the network, it is necessary to implement algorithms for identification and access authorization to the inertia element settings;

- controller-user communication via an external server. Both the controller and the user are able to view and modify data present on the server. The data exchange with the server is realized via a Web service by calling HTTP GET or HTTP POST methods. Thus, there is no need to unblock ports, and the communication takes place via port 80 available for HTTP as a standard. One server can provide communication for many controllers, distinguishing them by, e.g., a network adapter MAC address transmitted with the use of the GET method. Moreover, this solution enables transmission encryption using the SSL protocol. A disadvantage of this approach to the controller-user communication is the need to use an additional server. The server replies to queries and never initiates connections. As a result, the controller does not have to be assigned a public IP address and may use an IP address from the router pool. Moreover, it is possible to communicate with the controller from many computers.

This article discusses and presents implementations of the above-mentioned controller-user communication methods via the Internet [2]. Other methods (e.g. User Datagram Protocol (UDP) or Transmission Control Protocol (TCP)), which, for correct functioning, require router settings modification, e.g., by allowing to support ports other than port 80 , have not been discussed. 


\section{Flywheel energy storage system}

The flywheel energy storage [1] is based on a three-phase BLDCPM [3-9] motor with maximum rotational speed of $42,000 \mathrm{rpm}$. The motor electrical power equals $2 \mathrm{~kW}$. The moment of inertia of the inertia element rotating parts has been calculated at $45 \cdot 10^{-3}\left[\mathrm{~kg} \cdot \mathrm{m}^{2}\right]$, which at maximum speed enables to store energy of 435,549 [kJ] $=1.21[\mathrm{kWh}]$. The converter supporting the inverter is supplied from a one-phase bidirectional PFC $[9,10]$ power supply with unipolar modulation. Figure 1 shows the power circuit diagram of the converters. The current and voltage measurement paths are separated with the use of LEM transducers.

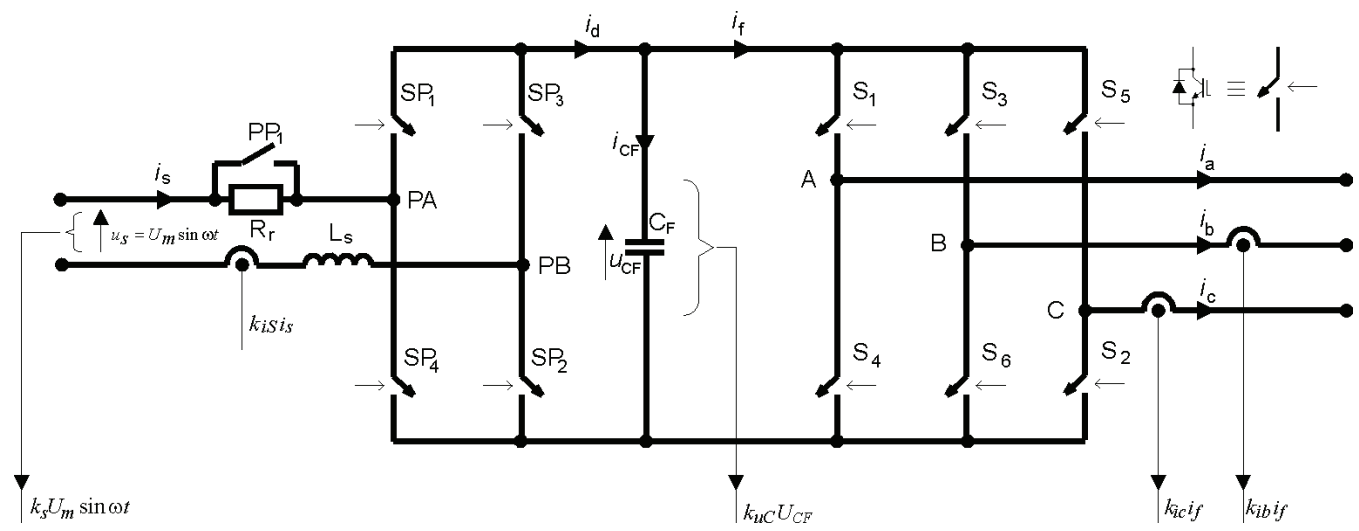

Fig. 1. Flywheel energy storage power circuit diagram

\section{Front-end rectifier}

The function of the power supply control system is to stabilize the set value of the voltage on the output capacitor with bidirectional energy transfer and to force the current to flow through the feed line, cophasal with the voltage and in a shape corresponding to this voltage. The power supply is to operate with a constant transistor switching frequency. Figure 2 shows the control system diagram fulfilling the set requirements.

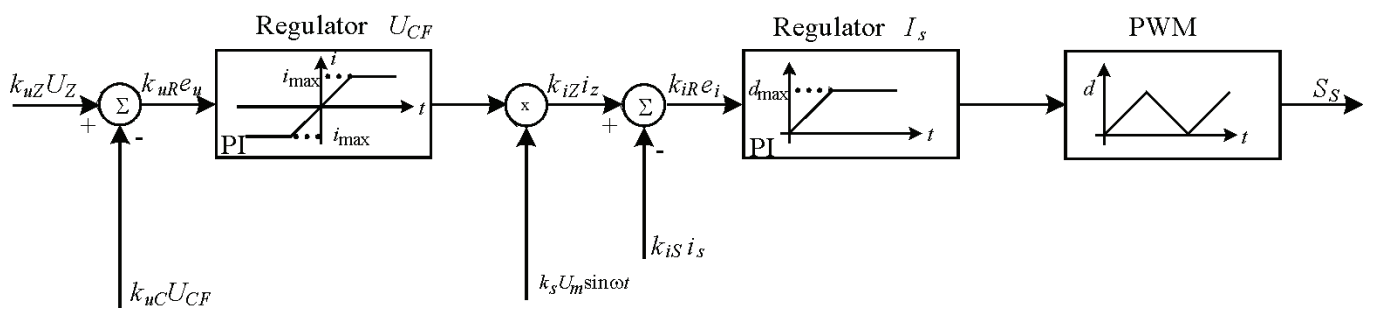

Fig. 2. Single-phase power supply control system diagram 
A signal proportional to the control error of the constant voltage $\left(e_{u}\right)$ is input to the voltage proportional integral regulator input. The voltage regulator output signal is proportional to the source current amplitude set in the system, multiplied by the signal proportional to the momentary value of the supply voltage $\left(k_{s} U_{m} \sin \omega \mathrm{t}\right)$. The result of this multiplication is a signal proportional to the set source current, cophasal with the supply voltage. This signal is input to the adder determining the current control error $\left(e_{i}\right)$. This error is input to the PI current regulator. The regulator constraints do not allow to achieve incorrect values of the pulse duty cycle (greater than one). It is a constraint resulting from the operation area scope of the sawtooth generator. The current regulator output is proportional to the pulse duty cycle $D$.

The control system is blocked until the capacitor $C_{F}$ is charged to the voltage of approximately $0.95 U_{S \max }$ by the PFC system transistor diodes. The charging current is limited by the resistor $R_{r}$ short-circuited by a relay after the capacitor voltage reaches to value of approximately $0.95 U_{S \max }$. Figure 3 shows the feed line current during the system operation.

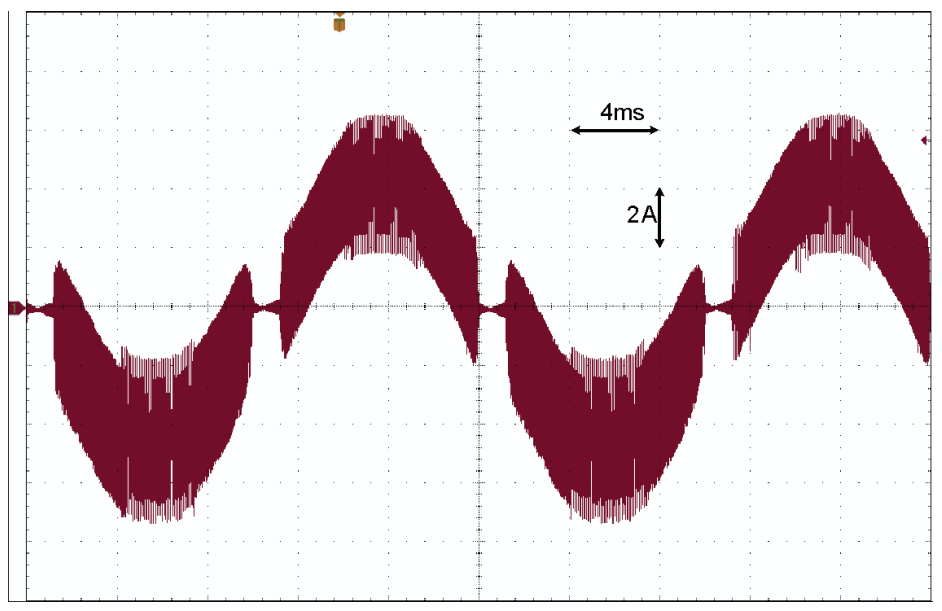

Fig. 3. Source current

\section{Motor control system}

Figure 4 shows the inverter control system diagram. Figure 4 assumes the following labels: $H_{A}, H_{B}, H_{C}$ - signals from the Hall effect sensor (determining the rotor position in relation to the stator), $M U X$ - multiplexer, $S A W$ - symmetrical sawtooth generator; $u_{\text {cap }}$ - voltage on the link circuit capacitor, $A B S$ - absolute value, $K S$ - sign comparator, $i_{x F}$ - current measured in the link circuit.

The converter controls the voltage on the link circuit capacitor. If the voltage drops below the assumed level, the speed of $1 / 3 \omega_{\max }$. is set to the control system. This results in a switch from motor operation to generator operation and conversion of mechanical energy into electrical energy. The control of the voltage on the capacitor is required also at the converter start. The inverter remains blocked until the proper startup of the network converter. The current storage rotational speed is determined on the basis of the Hall sensor signal frequency. 


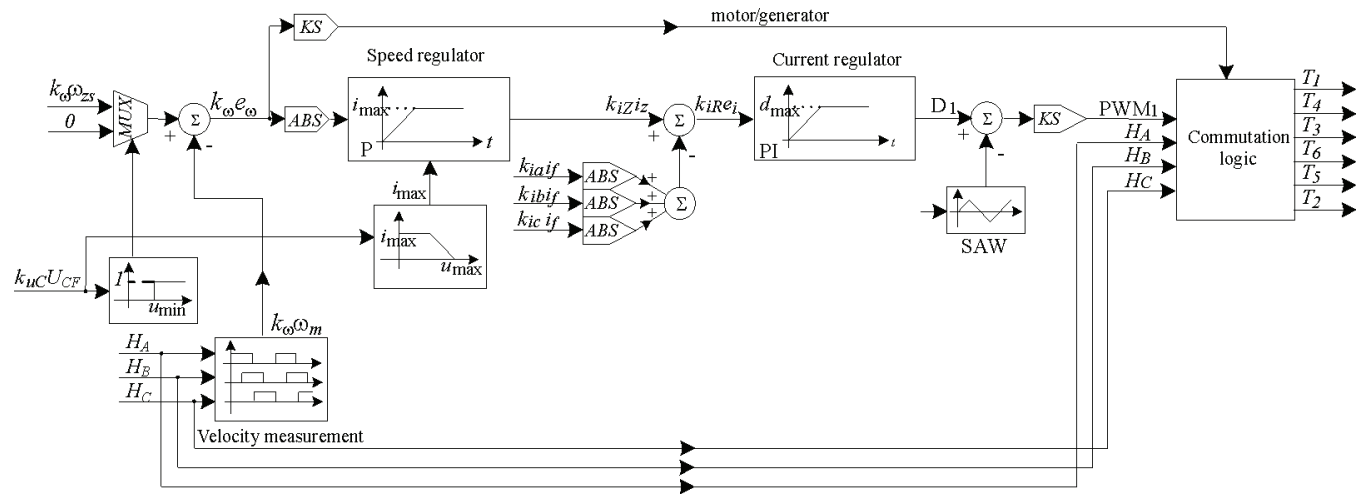

Fig. 4. Inverter control system diagram

Figure 5 shows motor phase current at the speed of 41,760 [rpm].

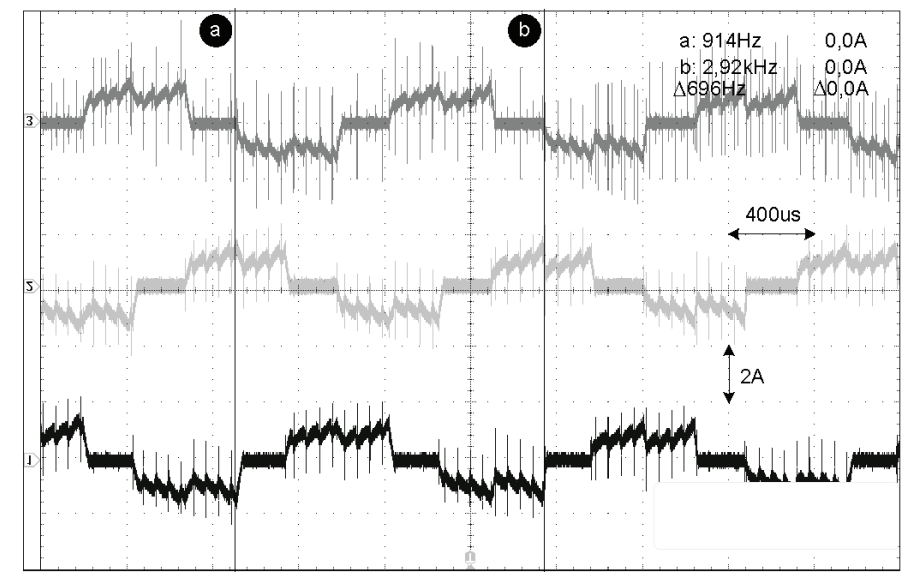

Fig. 5. Motor phase currents at the speed of $41,760 \mathrm{rpm}$

All elements of the control system using PFC power supply and the inverter (regulators, protections, startup sequence) were implemented in an Altera Cyclone III FPGA. A MAX1308 12-bit analog-to-digital converter (ADC) was used to acquire analog signals.

A distinctive feature of FPGAs is the capability of parallel processing. This property was used during the control system implementation and the converter adjustment. Owing to that, a structure of four independent regulators and separate overcurrent and overvoltage protections was implemented in one system. In the case of the converter control system, the concurrent operation of FPGA is desired and it facilitates the implementation, while the user interface implementation in systems operating in parallel is heavily obstructed. Thus, an FPGA implemented soft-core processor (NIOS II) is responsible for interactions with the user. Table 1 lists implementation methods of individual converter functions. 
Table 1. List of converter functions

\begin{tabular}{c|c|c}
\hline Item & Function & Implementation \\
\hline 1 & PFC control system & FPGA \\
\hline 2 & PFC system protections & FPGA \\
\hline 3 & inverter control system & FPGA \\
\hline 4 & inverter protections & FPGA \\
\hline 5 & system start & FPGA \\
\hline 6 & ADC system support & FPGA \\
\hline 7 & communication system support & NIOS II \\
\hline 8 & control buttons support & NIOS II \\
\hline 9 & measurement data registration & NIOS II \\
\hline
\end{tabular}

The FPGA used consists of 24,624 logic elements (approximately 20\% used in the project), 608,256 bytes of RAM (12\%) and 132 hardware multipliers (6\%). Table 2 presents the use of FPGA resources.

Table 2. List of converter functions

\begin{tabular}{c|c|c|c|c}
\hline Item & Function & Number of logic elements & Hardware multipliers & RAM (kB) \\
\hline 1 & PFC power supply & 1,020 & 4 & 0 \\
\hline 2 & Inverter & 1,704 & 4 & 0 \\
\hline 3 & $\begin{array}{c}\text { other functions } \\
\text { (e.g. AD interface) }\end{array}$ & 226 & 0 & 0 \\
\hline 4 & Nios II processor & 1,112 & 0 & 75,776 \\
\hline
\end{tabular}

The NIOS II processor implemented within FPGA structure has access to the control system data bus (measurement of currents, supply voltages and link circuit, motor rotation speed, set speed and information about possible errors). When a change of any monitored converter parameters is detected, the microprocessor sends it to the communication module.

\section{HTTP server implementation}

The HTTP server has been executed in a module equipped with a 16-bit microprocessor and the Ethernet physical layer controller, 10BASE-T compliant. The communication with the flywheel energy storage control system utilizes an RS485 interface and a MODBUS RTU protocol. In such a case, the system shown in Figure 6 functions as the master unit in the MODBUS network. Figure 6 shows a photograph of this system.

In order to facilitate the system configuration, it is equipped with a DHCP (Dynamic Host Configuration Protocol) support mechanism enabling to download the configuration settings from the server (in the case of operation within the assigned area of the Internet; see Fig. 7). 
Fig. 6. Photograph of the Internet communication module
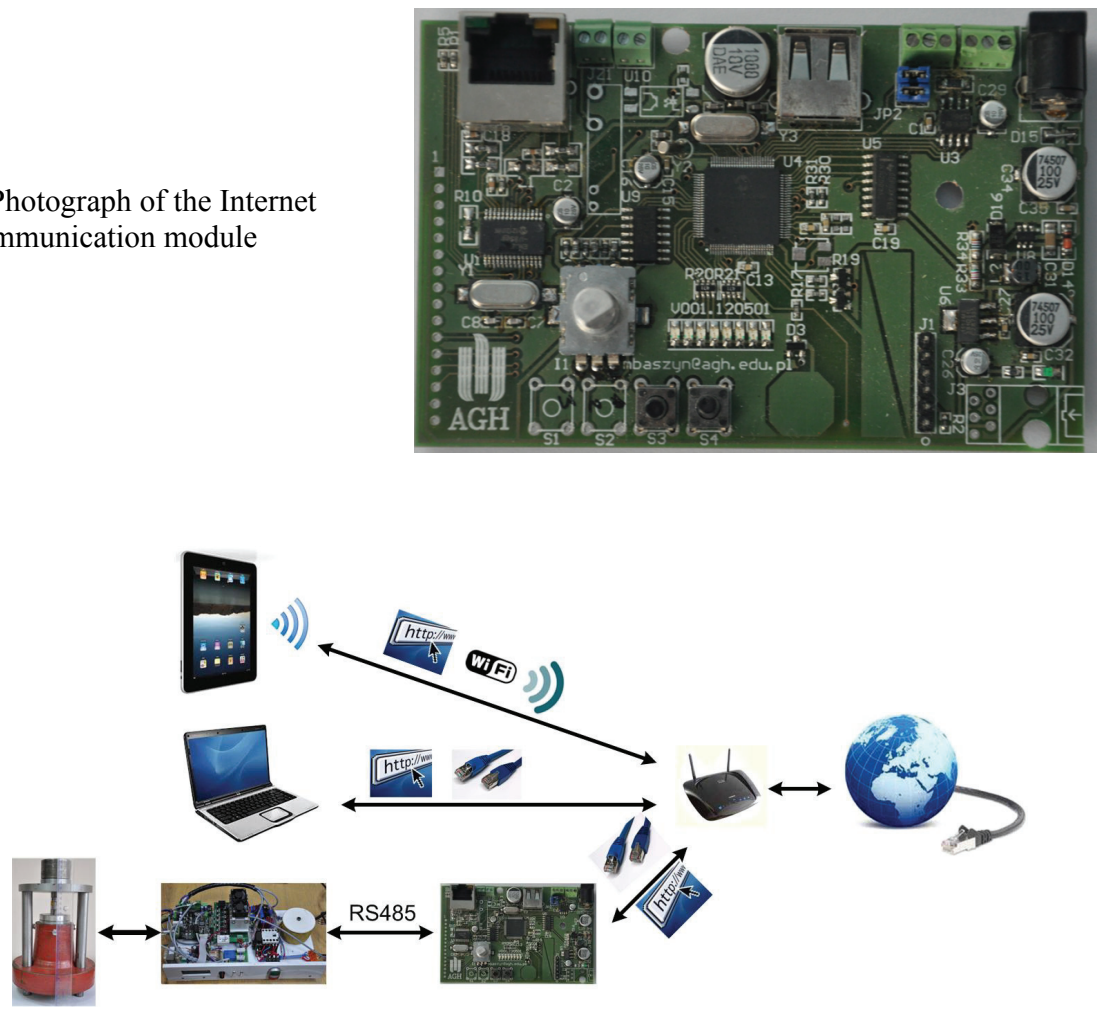

Fig. 7. Connection diagram of the system operating within the assigned area of the Internet

The module can also operate in the static settings mode (IP, DNS, subnet mask e.g. the system operating with a public IP address Fig. 8).

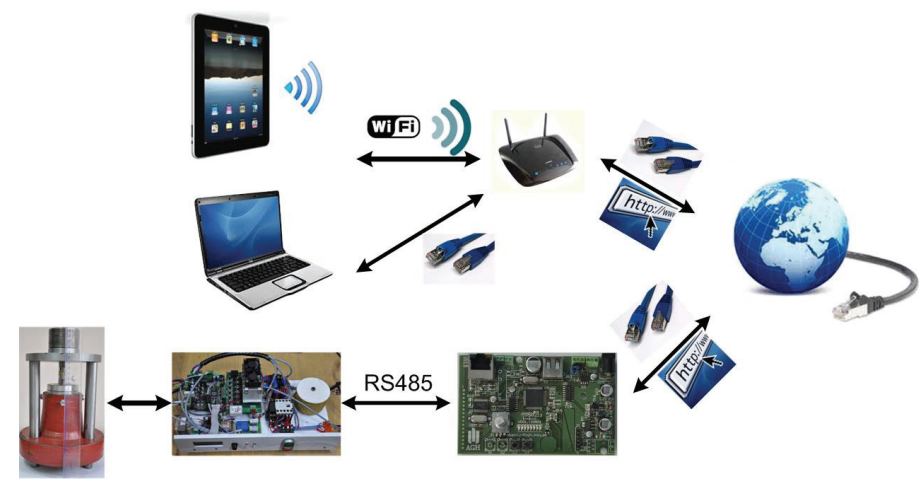

Fig. 8. Connection diagram of the system operating with a public IP address

In order to protect the control system against unauthorized access, when launching a web page in a web browser, a login window is displayed where the user name and the individual access code should be entered. This window is shown in Figure 9. 


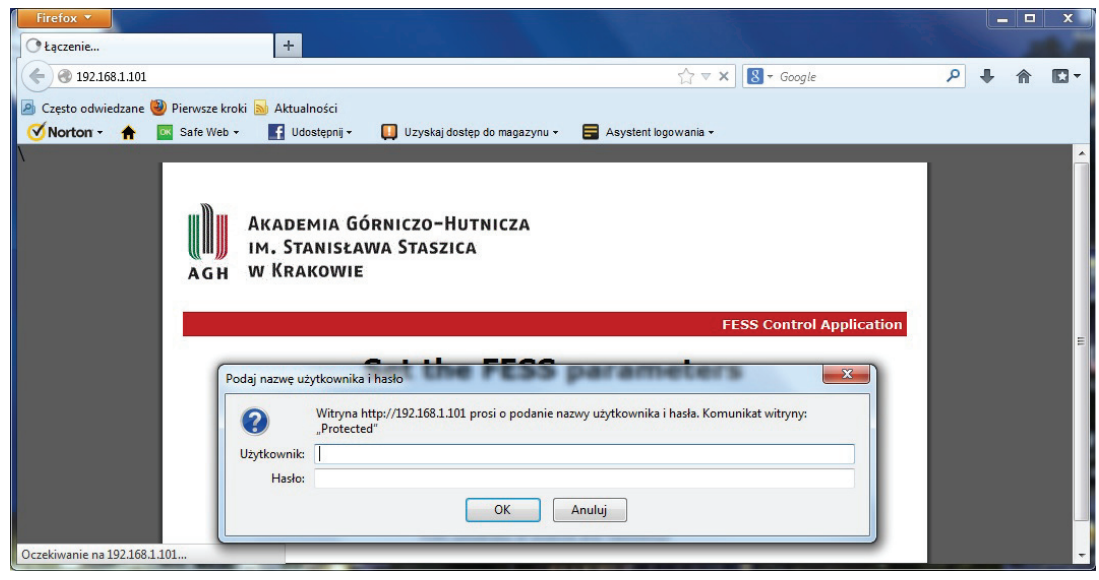

Fig. 9. HTTP server login window

After the correct login to the server, a window is displayed where storage parameters (set speed, start/stop, rotation direction) can be changed. The entered settings are confirmed by a call of the HTTP GET method from the browser level. It is executed by pressing the Save button. Figure 10 shows the view of this window.

The page shown in Figure 10 was developed in order to enable asynchronous data exchange between the user and the server without the need to reload the entire page (AJAX technique - Asynchronous JavaScript and XML). This solution enables the continuous updating of the storage rotational speed without the user interference.

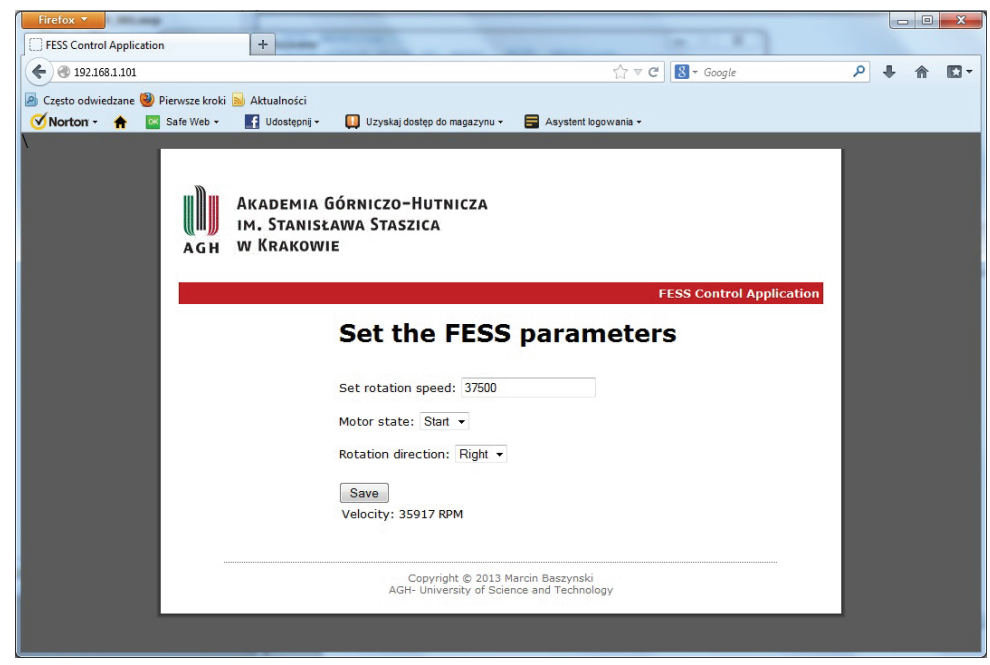

Fig. 10. Window enabling storage parameter change 


\section{Communications using Web Services}

A disadvantage of the HTTP server implementation in the controller communication is the access to the server limited to the assigned area of subnetwork (devices connected to one router) or the need to assign a public IP address to it. A solution devoid of these disadvantages is the data exchange between the user and the controller via Web Services. Figure 11 shows a diagram of this solution

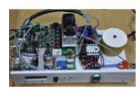

FESS

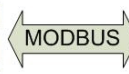
MODBUS

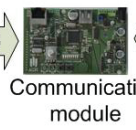

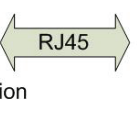

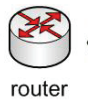

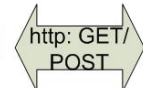

router POST
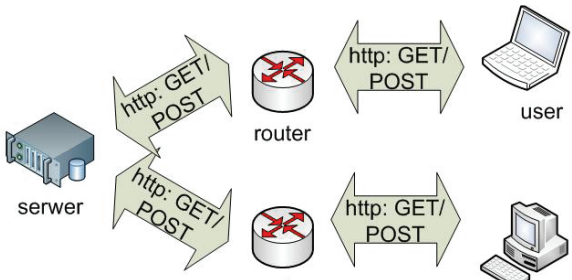

router user router

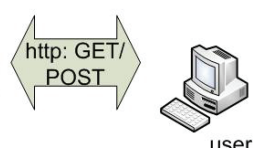

Fig. 11. Data exchange structure diagram with the use of Web Services

The data exchange between the user and the energy storage control system is realized via an external server (Fig. 11). The communication employs HTTP GET/POST queries supported by a Web Service. The controller and the user, independently of each other, initiate a connection with the server which functions as a database sharing information on request. The database stores information about the current storage operating parameters. Sending binary data with the use of the HTTP GET method requires their appropriate encoding, e.g., with the use of JSON (JavaScript Object Notation) or base64 format. After receiving the encoded data, the server decodes them to the binary format and saves them in the database. Status registers, such as start, motor stop, or set speed change can be changed both by the controller and the user.

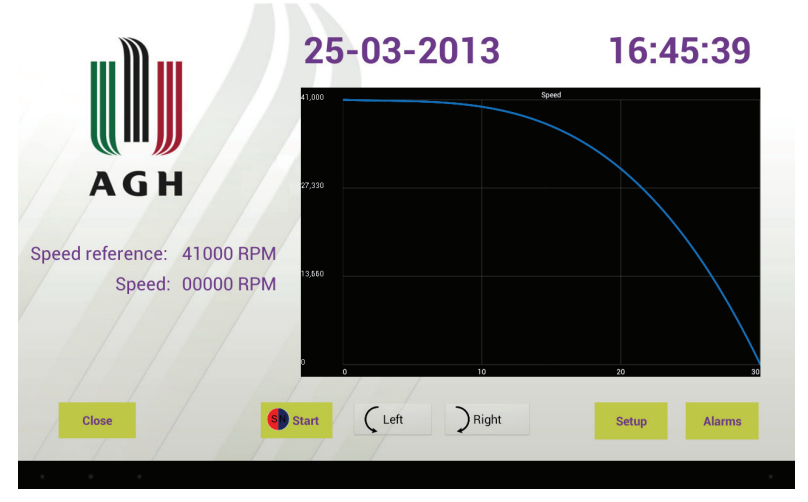

Fig. 12. Program view from the tablet controlling the drive operation

For a convenient server data access, an application was developed operating under the supervision of the Android operating system. It is responsible for the communication with the 
server and the energy storage operating parameters change. After receiving data from the server, the application displays them on the screen and saves them to an SD card if the program is operating in the recording mode. Saving the system operating parameters to the card enables their later analysis. The control program screen features buttons enabling to change the drive operating parameters. Figure 12 shows the control program screen view.

If the control program receives information about an error (e.g. overcurrent), an appropriate message is displayed on the screen (Fig. 13).

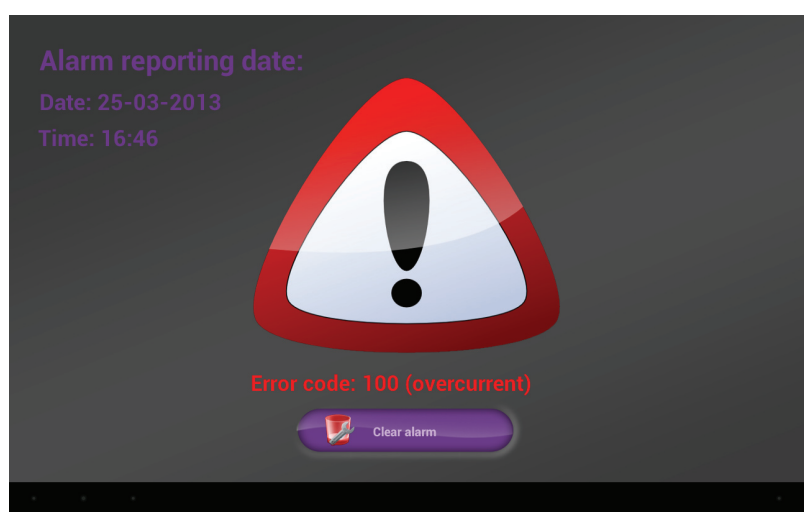

Fig. 13. Information screen view

If there is no Internet access, the application can make a connection from the Android system to the communication system via a USB link. In such a case, the communication module is recognized by the Android system as an external accessory with which there is a possibility of direct data exchange. Connecting the communication system to a device equipped with the Android operating system via a USB cable results in the automatic launching of the application shown in Figure 11, and switching it to the offline operating mode. The application remains fully functional, which enables the control of the flywheel energy storage operation.

In order to provide the user with the comfort of use, the application in the Android system is supported by three independent threads:

1) main thread - responsible for the interaction with the user, memory support, SD cards;

2) thread supporting the USB communication with the communication module;

3) thread supporting the Internet communication - responsible for the communication with the server; a data exchange with the server is initiated cyclically every 30 seconds.

\section{Summary}

The presented FESS control algorithm with Internet communication module was also tested on a FES system with power $35 \mathrm{~kW}$ and any disturbances in system operation were observed during this tests. Figure 14 shows motor phase current of the $35 \mathrm{~kW}$ FESS. 


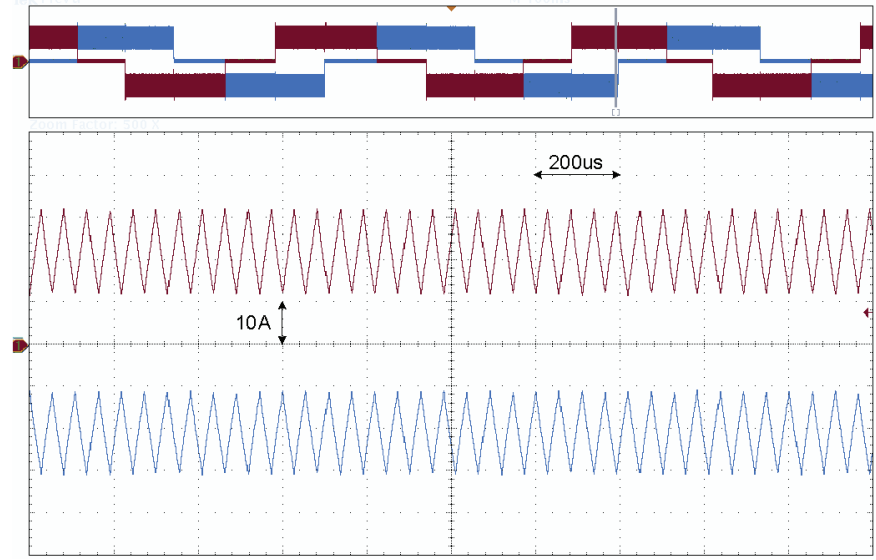

Fig. 14. Motor phase currents

The article discusses various methods of the power electronic system control via the Internet. An implementation of the flywheel energy storage control system has been presented, with an interface enabling remote control and measurement data archiving. This solution is based on widely available and inexpensive elements, which makes it very attractive for scientific and commercial applications.

\section{References}

[1] Piróg S., Baszyński M., Siostrzonek T., Energy Storage- The High-Speer Flywheel Energy Storage System. SCIYO, pp. 37-67 (2009).

[2] Sziebig G., Takarics B., Korondi P., Control of an Embedded System via Internet. Industrial Electronics, IEEE Transactions on 57(10): 3324-3333 (2010).

[3] Bernat J., Stępien S., Application of optimal current driver for the torque control of BLDC motor. Archives of Electrical Engineering 60(2): 149-158 (2011).

[4] Ciurys M., Dudzikowski I., Transients of electrical and mechanical quantities of a brushless DC motor - computations, measurements. Archives of Electrical Engineering 60(1): 23-34 (2011).

[5] Kaliappan E., Chellamuthu C., Modeling, simulation and experimental analysis of permanent magnet brushless DC motors for sensorless operations. Archives of Electrical Engineering 61(4): 499-515 (2012).

[6] Biskup T., Recovery braking of variable-structure electronic commutator for BLDC motor. Archives of Electrical Engineering 61(4): 579-596 (2012).

[7] Joice C.S., Paranjothi S.R., Kumar V.J.S, Digital Control Strategy for Four Quadrant Operation of Three Phase BLDC Motor With Load Variations. IEEE Trans. on Industrial Informatics 9(2): 974-982 (2013).

[8] Das P., Pahlevaninezhad M., Drobnik J. et al., A Nonlinear Controller Based on a Discrete Energy Function for an AC/DC Boost PFC Converter. IEEE Trans. on Industrial Application 28(12): 5458-5476 (2013).

[9] Kulikowski K., Modified algorithms of direct power control of AC/DC converter co-operating with the grid. Archives of Electrical Engineering 61(3): 373-388 (2012).

[10] Stupar A., Friedli T., Minibock J., Kolar J.W., Towards a 99\% Efficient Three-Phase Buck-Type PFC Rectifier for 400-V DC Distribution Systems. IEEE Trans. on Power Electronics 27(4): 1732-1744 (2012). 